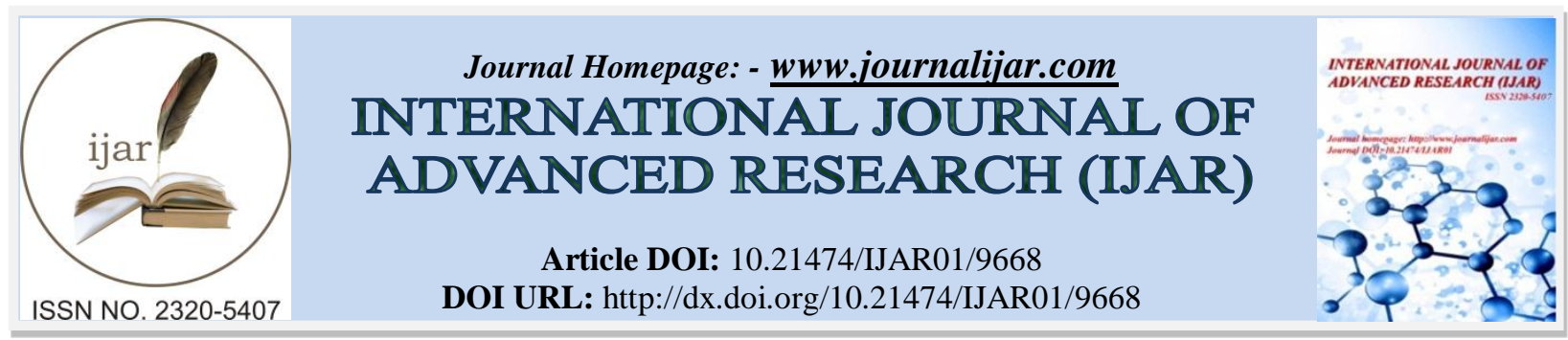

RESEARCH ARTICLE

\title{
EFFECTS OF MENTAL HEALTH (DEPRESSION, ANXIETY AND STRESS) ON RECOVERY PERIOD OF CHRONIC LOWER RESPIRATORY TRACT INFECTION (LRTI) PATIENTS.
}

\author{
Ankita Misra ,Dr. Manjiri Datar, Dr. Bhupendra Chaudhary, Ansh Chaudhary and Ayush Jain. \\ F-101, Nancy Lake Home Society, Katraj, Pune, Maharashtra.
}

\section{Manuscript Info}

Manuscript History

Received: 04 July 2019

Final Accepted: 06 August 2019

Published: September 2019

\section{Abstract}

\section{Introduction:-}

Although the very definition of 'health' speaks of physical as well as mental well-being, more often than not, the latter is brushed off as unimportant. An integral part of our humane existence is still glossed over by many a great medical practitioner as a work of fiction, receiving little attention within existing literature. This study takes up the mantle to bring this issue of the physiological effects of psychological disorders, into the limelight it deserves.

Anxiety triggers the "flight or fight" response, releasing chemicals and hormones like adrenaline into our system. According to a Harvard Medical School study, an increased rate of panic and anxiety attacks have been encountered in the US given how anxiety increases breathing rate, leading to increased cellular oxygen levels, leading to a feeling of breathlessness, dizziness and nausea. This can also lead to a prolonged recovery period for patients in later stages of the illness given how anxiety could slow the process of recovery by triggering adrenaline and sending the body in an artificial shock and thus, in need of more oxygen. This process gets triggered cyclically due to the anxiety disorder, thereby sufficiently damaging the sufferer's general physiological health.[1]

Stress is a reaction to a stimulus disturbing physical or mental equilibrium. Stressful events trigger the "fight-orflight" response, causing hormones such as adrenaline and cortisol to surge through the body. Causative agents of stress or stress itself cause potent exacerbation of respiratory infections. Not only does stress affect susceptibility to infections based on it's inter-relationship with the immune system but increased evidence is also being gathered that respiratory pathogens are equally responsive to the hormonal output of stress.[2]

Psychological disorders left undiagnosed and untreated tend to worsen the general health of a person and hamper the improvement of a physiological ailment. There is further requirement to substantiate psychological disorders as the threat they are to physiological health [3].

\section{Methodology:-}

Study Setting:

University Medical College and Tertiary Care Hospital

Study Design:

Correlational Cross-sectional Study

Corresponding Author:-Ankita Misra.

Address:-F-101, Nancy Lake Home Society, Katraj, Pune, Maharashtra. 


\section{Sample Size Estimation:}

50 patients

\section{Inclusion Criteria:}

Hospitalized patients above the age of 18 admitted more than once in the previous one-year time frame or who have had exacerbations of their presenting lower respiratory tract infection (Bronchitis, Pneumonia etc.) that lasted up to 21 days, associated with common comorbidities, such as Chronic Obstructive Pulmonary Disease, Interstitial Lung Disease, Congestive Cardiac Failure, Diabetes and Hypertension.

\section{Exclusion Criteria:}

Children and Adolescents below the age of 18, patients with psychoses or any mental retardation.

Collection of data was done in 2 parts- the socio-demographic details of the patient were collected as per a standardized proforma, which included data regarding number of hospital admissions in past one-year and current period of hospitalization as well as an author-designed structured questionnaire to assess the scale of satisfaction towards the perceived social support system of the patient during the period of their illness. The Depression, Anxiety and Stress Scale (DASS) questionnaire to assess levels of depression, anxiety and stress symptoms in the patients.

The author-designed structured questionnaire to assess levels of satisfaction with respect to perceived social support system was on the basis of five parameters and the patient was asked whether each parameter was met or not. These parameters are as follows-

1. Amount of time support system spent in the hospital with them (increased and undivided attention)

2. Empathetic nature of perceived social support (and not sympathetic)

3. Positivity, hope and comfort provided

4. Serving as a bridge of communication between doctor and patient

5. Fulfilling responsibilities on patient's behalf

The 3-point scale of satisfaction is as follows-

4-5 parameters out of 5 met $=$ Good

3 out of 5 parameters met $=$ Satisfactory/ Neutral

1-2 parameters out of 5 met $=$ Not Satisfactory

The DASS- Depression, Anxiety and Stress Scale is a set of three self-report scales designed to measure and quantify the negative emotional states of depression, anxiety and stress.

Each scale contains 14 items, divided into subscales of 2-5 items with similar content. The Depression scale assesses dysphoria, hopelessness, devaluation of life, self-deprecation, lack of interest/involvement, anhedonia, and inertia. The Anxiety scale assesses autonomic arousal, skeletal muscle effects, situational anxiety, and subjective experience of anxious affect. The Stress scale is sensitive to levels of chronic non-specific arousal. It assesses difficulty relaxing, nervous arousal, and being easily upset/agitated, irritable/over-reactive and impatient. Subjects use 4-point severity/frequency scales to rate the extents to which they experienced each state over the duration of illness. Scores are calculated by summing the scores for the relevant items. Resultant scores of the DASS are obtained as follows-

Stress :

$\begin{array}{lll}\text { Normal } & 0-10 & \\ \text { Mild } & & 11-18 \\ \text { Moderate } & & 19-26 \\ \text { Severe } & 27-34 & \\ \text { Extremely severe } & 35-42 & \\ & \end{array}$

Anxiety :

Normal $\quad 0-6$

Mild

7-9

Moderate

10-14

Severe 15-19

Depression :

Extremely severe 20-42

Normal

0-9

Mild

10-12 
Moderate

Severe

Extremely severe
$13-20$

21-27

28-42

The scales of the DASS have been shown to have high internal consistency and to yield pertinent discriminations in a variety of environments, especially clinical.

The questionnaire was administered orally one-on-one with patients in Hindi if therein lied their comfort, and statements were explained if unclear.

\section{Observations and Results:-}

The Chi-square test was used to statistically analyze the relationships between depression, stress and anxiety with different variables- socio-demographic predictors, perceived social support, period of hospitalization and duration of illness and $\mathrm{p} \leq 0.05$ was considered as significant.

The sample size comprised of 50 patients across 5 age groups- I, $\leq 30$ years of age amounting to $8 \%$ of the sample, II comprising of 31-40 years (22\%), III- 41-50 years (12\%), IV- 51-60 years of age (16\%) and finally, the most common age group (V) encompassing patients 61 years of age and above, were $42 \%$ of the sample. $58 \%$ of the participants were female, $42 \%$ male, with $64 \%$ of the participants belonging to middle-class background, $26 \%$ from above poverty level and $10 \%$ below poverty level. $96 \%$ of the participants were married.

In the present study, it was observed that $82 \%$ of the patients tested positive for stress- $20 \%$ participants had mild, $32 \%$ had moderate, $22 \%$ had severe and $8 \%$ had extremely severe stress. Of all the participants that tested positive for anxiety- $16 \%$ participants tested for mild, $38 \%$ participants tested for moderate, $22 \%$ for severe and $20 \%$ for extremely severe anxiety. $52 \%$ of the participants tested positive for depression- $24 \%$ for mild, $18 \%$ for moderate, $6 \%$ for severe and $4 \%$ for extremely severe depression. Prevalence rates for mild to moderate stress was $52 \%$ and for severe to extremely severe, $30 \%$. Prevalence rates for mild to moderate anxiety was $54 \%$ and for severe to extremely severe, $42 \%$. Prevalence rates for mild to moderate depression was $42 \%$ and for severe to extremely severe, $10 \%$.

Scale of satisfaction of perceived support system during illness varied from $38 \%$ subjects finding their perceived social support system as 'Good', 44\% as 'Satisfactory' or 'Average' and finally $18 \%$ as 'Not Satisfactory'.

As predictors of psychological and physiological well-being, there was no significant variation in the prevalence of depression $(\mathrm{p}=0.351)$, anxiety $(\mathrm{p}=0.615)$ or stress $(\mathrm{p}=0.677)$ on the basis of gender or between different age groups with depression $(p=0.652)$, anxiety $(p=0.274)$ or stress $(p=0.535)$.

There was a significant relationship $(\mathbf{p = 0 . 0 0 9 )}$ in prevalence of different degrees of depression as per different socio-economic backgrounds. Out of the $64 \%$ of the participants belonging to the middle-class background, $36 \%$ tested negatively for presence of depression, $24 \%$ for mild to moderate depression and $4 \%$ for severe. Of the $26 \%$ of the participants that belonged from above poverty level, $12 \%$ tested negative for depression, $12 \%$ for mild to moderate depression and $2 \%$ for extremely severe depression. $10 \%$ of the participants belonged to below poverty level socio-economic background and the entire $10 \%$ tested positive for depression, ranging from moderate to extremely severe depression- $6 \%$ for moderate, and $2 \%$ each for severe and extremely severe depression.

A significant correlation was found between the 3-point scale of satisfaction of perceived support system and levels of depression, anxiety and stress $(\mathbf{p}<\mathbf{0 . 0 0 1}$ for anxiety and depression and $\mathbf{p}=\mathbf{0 . 0 4 0}$ for anxiety).

19 out of 50 participants $(38 \%)$ were well satisfied with their perceived support system (Grade 1 on the scale of satisfaction). It was observed that $47.37 \%$ of these 19 participants ( 9 participants) tested negative for stress, $10.5 \%$ negative for anxiety and $84.2 \%$ tested negative for depression. $47.37 \%$ tested positive for mild to moderate stress, $68.4 \%$ tested positive for mild to moderate anxiety and $15 \%$ tested positive for mild to moderate depression. Only 1 participant tested positive for severe stress, 2 for severe anxiety and none for severe depression.

22 out of 50 participants $(44 \%)$ were only partially satisfied with their social support system. All of these participants tested positive for different levels of stress and anxiety. $18.18 \%$ of these 22 participants tested positive 
for mild stress and $13.65 \%$ for mild anxiety. $45.45 \%$ participants had moderate stress and moderate anxiety. $31.82 \%$ subjects had severe stress and $27.27 \%$ had severe anxiety. $4.5 \%$ had extremely severe stress and $13.64 \%$ had extremely severe anxiety. $27.27 \%$ participants had mild depression, $27.27 \%$ had moderate, $4.5 \%$ had severe and $4.5 \%$ had extremely severe depression.

9 out of 50 participants (18\%) were not satisfied with their social support system. All these remaining $18 \%$ subjects scored positive for moderate to extremely severe stress and anxiety. $44.44 \%$ of the subjects had mild depression. While $22.22 \%$ subjects had moderate depression, the same percentage prevailed for subjects with severe depression. $11.11 \%$ of these 9 participants had extremely severe-depression. (Table 1.)

It is inferred that those participants with a better level of satisfaction with their perceived social support system, had lesser levels of stress, anxiety and depression than those that were partially dissatisfied or completely dissatisfied with the same.

Table 2 represents a correlation between depression, stress and anxiety with duration of illness. While a significant relationship was not observed between levels of anxiety (0.135) and stress (0.305) with duration of illness, a significant relationship $(\mathbf{p}=\mathbf{0 . 0 0 3})$ was observed between depression and duration of illness suffered by the participants. 33 participants with period of illness $\leq 1$ year $(66 \%)$, exhibited lower scores on the DASS, ranging from normal (54.5\% of these 33 participants), mild (27.27\%) to moderate depression (18.18\%). None of these participants had severe or extremely depression. $16 \%$ of the 50 participants had been suffering from their chronic LRTI for 1-2 years, of these $16 \%$, half tested normal but $37.5 \%$ ( $6 \%$ of the total sample size) tested for moderate depression and $12.5 \%$ for severe. None of these participants tested positive for extremely severe depression either. Finally, the duration of illness of the final $18 \%$ of the participants was for a period of $>2$ years, and their scores ranged from normal to extremely severe i.e. from one end of the spectrum to another. Of these 9 participants, $22 \%$ tested normal, $33 \%$ for mild depression and $22 \%$ severe and extremely severe depression each. Hence inferred that participants that exhibited lower scores on the DASS had a lesser duration of illness and vice versa.

There was no significant relationship observed between number of times of hospitalization in the past one year of illness and levels of anxiety, depression and stress $(\mathrm{p}=0.75)$

\section{Discussion:-}

This study observed that there was a higher prevalence rate of stress and anxiety in the sample, as compared to depression. In a systematic review of studies that reported the prevalence of the same in patients of COPD by Wilgoss and Yohannes, it was observed that in all statistically relevant studies, though there was a high prevalence of anxiety and depression that was noted in patients with COPD, the occurrence of anxiety was higher than that of depression.[4]

There was a significant relationship $(\mathrm{p}=0.009)$ in prevalence of different degrees of depression as per different socioeconomic backgrounds. It was observed that socio-economic background of an individual has a significant and directly proportionate impact on that individual's depressive tendencies. The findings from this study simply reinforces findings obtained from previous studies as well. A meta-analysis on socioeconomic inequalities in depression by Lorant et al revealed conclusively that lower socio-economic classes had higher odds of being depressed.[5]

In the present study, a significant correlation was found between the 3-point scale of satisfaction of perceived support system and levels of depression, anxiety and stress $(\mathrm{p}<0.001$ for anxiety and depression and $\mathrm{p}=0.040$ for anxiety). Participants well satisfied with their perceived social support system had lower prevalence of severe and extremely severe anxiety, none for severe or extremely severe stress or depression, as compared to those participants that were either partially or not satisfied. Lyrra and Heikkinen examined the effect of perceived social support on mortality, arriving at the conclusion that those individuals that had lowest degree of perceived social support, comprising infrequent experiences of reassurance of worth, emotional closeness, sense of belonging and opportunity for nurturance, had higher risk of mortality.[6] Curtis et al showed that coping strategies like emotional venting, are shown to have a positive effect on the health status of the individual[7]. The direct impact on depression, anxiety and stress by perceived social support, which in turn has an effect on healthy functioning of an individual, was also investigated in a study by Chen et al [8]. 
The current study's hallmark finding was that a significant relationship $(\mathrm{p}=0.003)$ was observed between depression and duration of illness suffered by the participants. Those participants that had a duration of illness $\leq 1$ year $(66 \%)$, exhibited lower scores on the DASS, ranging from normal (54.5\% of these 33 participants), mild (27.27\%) to moderate depression (18.18\%). None of these participants had severe or extremely depression. Meanwhile on the other end of the spectrum, those participants that had a duration of illness of greater than 1-2 years, had scores ranging from normal to extremely severe. Of these 9 participants, $22 \%$ tested normal, $33 \%$ for mild depression and $22 \%$ severe and extremely severe depression each. Therefore, Participants that exhibited lower scores on the DASS had a lesser duration of illness and vice versa.

Directly reinforcing the results of our study, an epidemiological population-based study of major relevance due to its sample size- (14364) by Kao et al, investigated correlation poor clinical outcomes of pneumonia patients with DD and discovered that patients with DD had significantly higher probability of ICU admission, need for mechanical ventilation and in-hospital death than patients without DD.[9] The correlation of depressive disorders and impact of the same on duration of illness was also demonstrated by Laurin et al which assessed the impact of psychiatric disorders on exacerbations of COPD, inferring that those with anxiety or depressive disorders were at a significantly higher risk of exacerbations and recurrence annually.[10] Additionally, supported by $\mathrm{Ng}$ et al, which discovered that comorbid depressive symptoms in patients with COPD are associated with longer recovery period.[11]

While a significant relationship between depression and duration of illness was observed in our study, a significant relationship was not observed between levels of anxiety (0.135) and stress (0.305) with duration of illness.

An explanation for why stress was not significantly associated with duration of illness in chronically ill patients can be postulated from a study by Medinas-Amorós et al, discovering that for chronically ill COPD patients, hospitalization was a seemingly less stressful event due to previous exposure to the same. An account so as to why anxiety was not found to be associated with duration of illness significantly, can be deduced from a study conducted by Fan et al where findings stated that anxiety was not associated with hospitalization or mortality. Depression was associated with increased risk for 3-year mortality but not 1-year mortality or hospitalization. This is possibly due to the fact that anxiety is more an acute response to a stressor than chronic, and over time, as stated by MedinasAmorós et al, over time the individual adapts to this stressor due to previous exposure. On the other hand, depression itself being a chronic disease developing over time, is an additional and more debilitating burden to the immune system and overall health status.[12-13]

$\mathrm{Xu}$ et al investigated the independent effect of depression and anxiety on COPD exacerbations and hospitalizations, discovered that there was a possible and significant cause-effect relationship of depression and COPD exacerbations, hospitalizations and health status (improvement or decline), but not with anxiety.[14]

\section{Conclusion:-}

Depression, stress and anxiety have negatively co-dependent relationships with chronic LRTIs, resulting in a vicious cycle with one amounting to an increment in the other, consecutively causing deterioration in the health status of the patient.

\section{Conflict of Interests}

The author(s) declare that there is no conflict of interest.

\section{Ethics}

There is no ethical violation in the conduction of the study and it has been approved by the ethical committee of the University.

\section{Funding}

The proposal was selected for the ICMR-STS 2018 Grant.

Table 1:-Correlation between DASS Scale and Scale of Satisfaction of Perceived Social Support SCALE OF SATISFACTION OF PERCEIVED SOCIAL SUPPORT 


\begin{tabular}{|c|c|c|c|c|c|c|c|c|c|c|c|c|c|c|c|c|}
\hline $\begin{array}{l}\text { PSS } \\
\text { n1= } \\
\text { n2=22, } \\
\text { n3=9 }\end{array}$ & 1 & 2 & 3 & 1 & 2 & 3 & 1 & 2 & 3 & 1 & 2 & 3 & 1 & 2 & 3 & P VALUE \\
\hline DASS & \multicolumn{3}{|c|}{ NORMAL } & \multicolumn{3}{|c|}{ MILD } & \multicolumn{3}{|c|}{ MODERATE } & \multicolumn{3}{|c|}{ SEVERE } & \multicolumn{2}{|c|}{$\begin{array}{l}\text { EXTREME } \\
\text {-LY } \\
\text { SEVERE }\end{array}$} & & \\
\hline $\begin{array}{l}\text { STRESS } \\
\text { SCORE } \\
\text { GROUP }\end{array}$ & 9 & 0 & 0 & 6 & 4 & 0 & 3 & 10 & 3 & 1 & 7 & 3 & 0 & 1 & 3 & $<0.001$ \\
\hline $\begin{array}{l}\text { ANXIETY } \\
\text { SCORE } \\
\text { GROUP }\end{array}$ & 2 & 0 & 0 & 5 & 3 & 0 & 8 & 10 & 1 & 2 & 6 & 3 & 2 & 3 & 5 & 0.040 \\
\hline $\begin{array}{l}\text { DEPRESSION } \\
\text { SCORE } \\
\text { GROUP }\end{array}$ & 16 & 8 & 0 & 2 & 6 & 4 & 1 & 6 & 2 & 0 & 1 & 2 & 0 & 1 & 1 & $<0.001$ \\
\hline
\end{tabular}

Key: Participants grouped ( $\mathrm{n}=$ number of participants in total in the group) according to their response to Scale of Satisfaction of Perceived Social Support, where-

1 = Good, where $n=19,2=$ Satisfactory/Neutral, where $n=22$ and $3=$ Not Satisfactory, where $n=9$.

Table No. 2:-Correlation of DASS Scale with Duration of Illness

\begin{tabular}{|c|c|c|c|c|c|c|c|c|c|c|c|c|c|c|c|c|}
\hline & \multicolumn{16}{|c|}{ DURATION OF ILLNESS } \\
\hline $\begin{array}{l}\mathrm{n} 1=33, \mathrm{n} 2=8, \\
\mathrm{n} 3=9\end{array}$ & 1 & 2 & 3 & 1 & 2 & 3 & 1 & 2 & 3 & 1 & 2 & 3 & 1 & 2 & 3 & P VALUE \\
\hline DASS & \multicolumn{3}{|c|}{ NORMAL } & \multicolumn{3}{|c|}{ MILD } & \multicolumn{3}{|c|}{ MODERATE } & \multicolumn{3}{|c|}{ SEVERE } & \multicolumn{2}{|c|}{$\begin{array}{l}\text { EXTREME } \\
\text {-LY } \\
\text { SEVERE }\end{array}$} & & \\
\hline $\begin{array}{l}\text { STRESS } \\
\text { SCORE } \\
\text { GROUP }\end{array}$ & 8 & 0 & 1 & 7 & 1 & 2 & 10 & 5 & 1 & 6 & 2 & 3 & 2 & 0 & 2 & 0.305 \\
\hline $\begin{array}{l}\text { ANXIETY } \\
\text { SCORE } \\
\text { GROUP }\end{array}$ & 2 & 0 & 0 & 7 & 1 & 0 & 14 & 2 & 3 & 6 & 4 & 1 & 4 & 1 & 6 & 0.135 \\
\hline
\end{tabular}




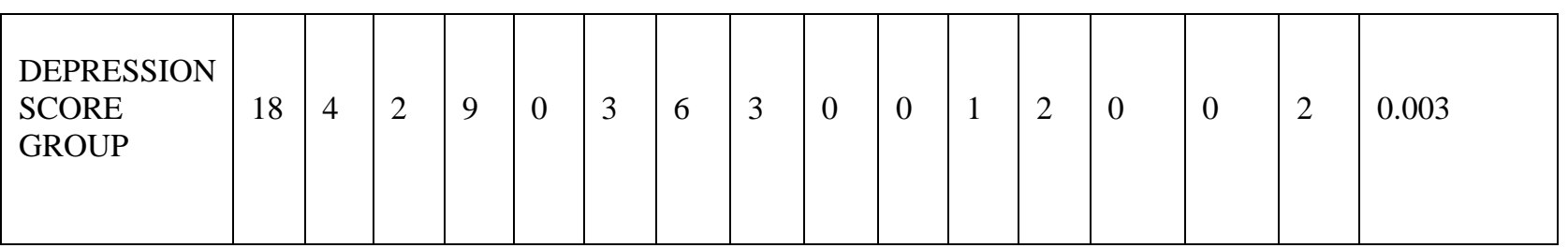

Key: Participants grouped ( $\mathrm{n}=$ number of participants in total in the group) according to their duration of illness, where-

$1=\leq 1$ year, where $n=33,2=1-2$ years, where $n=8$ and $3=>2$ years, where $n=9$.

\section{References:-}

1. Kozlowska K, Walker P, McLean L, Carrive P. Fear and the Defense Cascade: Clinical Implications and Management. Harvard Review of Psychiatry 2015;23(4):263-287.

2. Stover CM. Mechanisms of Stress-Mediated Modulation of Upper and Lower Respiratory Tract Infections. Adv Exp Med Biol. 2016; 874:215-23.

3. Voltmer E, Kieschke U, Schwappach DL, Wirsching M, Spahn C. Psychosocial health risk factors and resources of medical students and physicians: a cross-sectional study. BMC Med Educ. 2008 Oct 2; 8:46.

4. Willgoss TG, Yohannes AM. Anxiety disorders in patients with COPD: a systematic review. Respiratory Care. 2013 May;58(5):858-66.

5. Lorant V, Deliège D, Eaton W, Robert A, Philippot P, Ansseau M. Socioeconomic inequalities in depression: a meta-analysis. Am J Epidemiol. 2003 Jan 15; 157(2):98-112

6. Lyyra TM, Heikkinen RL. Perceived social support and mortality in older people. The Journal of Gerontology: Psychological Sciences Series B. 2006 May;61(3): S147-52.

7. Ruth Curtis, AnnMarie Groarke, Robert Coughlan \& Amina Gsel. The influence of disease severity, perceived stress, social support and coping in patients with chronic illness: A 1 year follow up Psychology, Health \& Medicine Journal, Volume 9, 2004 - Issue 4, Pages 456-475.

8. Chen YJ, Narsavage GL. Factors related to chronic obstructive pulmonary disease readmission in Taiwan. Western Journal of Nursing Research, 2006 Feb;28(1): Pages 105-24.

9. Li-Ting Kao, Shih-Ping Liu, Herng-Ching Lin, Hsin-Chien Lee, Ming-Chieh Tsai and Shiu-Dong Chung (2014). Poor Clinical Outcomes among Pneumonia Patients with Depressive Disorder. PLoS One Journal. 2014; $9(12)$.

10. Laurin C, Labrecque M, Dupuis G, Bacon S, Cartier A, Lavoie K. Chronic obstructive pulmonary disease patients with psychiatric disorders are at greater risk of exacerbations. Psychosomatic Medicine Journal. 2009 Jul;71(6):667-74.

11. Ng TP, Niti M, Tan WC, Cao Z, Ong KC, Eng P. Depressive symptoms and chronic obstructive pulmonary disease: effect on mortality, hospital readmission, symptom burden, functional status, and quality of life. Archives of Internal Medicine, 2007 Jan 8;167(1):60-7.

12. Magdalena Medinas-Amorós, Juan José Montaño-Moreno, Maria José Centeno-Flores, Victoria Ferrer-Pérez, Feliu Renom-Sotorra, Belen Martín-López and Catalina Alorda-Quetglas. Stress associated with hospitalization in patients with COPD: the role of social support and health related quality of life. Multidisciplinary Respiratory Medicine Journal. 2012 Dec 10;7(1):51.

13. Fan VS, Ramsey SD, Giardino ND, Make BJ, Emery CF, Diaz PT, Benditt JO, Mosenifar Z, McKenna R Jr, Curtis JL, Fishman AP, Martinez FJ. National Emphysema Treatment Trial (NETT) Research Group. Sex, depression, and risk of hospitalization and mortality in chronic obstructive pulmonary disease. Archives of Internal Medicine Journal, 2007, Nov 26;167(21):2345-53.

14. Xu W, Collet JP, Shapiro S, Lin Y, Yang T, Platt RW, Wang C, Bourbeau J. Independent effect of depression and anxiety on chronic obstructive pulmonary disease exacerbations and hospitalizations. American Journal of Respiratory and Critical Care Medicine, 2008, Nov 1;178(9):913-20. 